\title{
Analysis of Geomagnetic Storms Using Wavelet Transforms
}

\section{B. Adhikari, R. Khatiwada and N. P. Chapagain}

Journal of Nepal Physical Society

Volume 4, Issue 1, February 2017

ISSN : 2392-473X

Editors:

Dr. Gopi Chandra Kaphle

Dr. Devendra Adhikari

Mr. Deependra Parajuli

JNPS, 4 (1), 119-124 (2017)

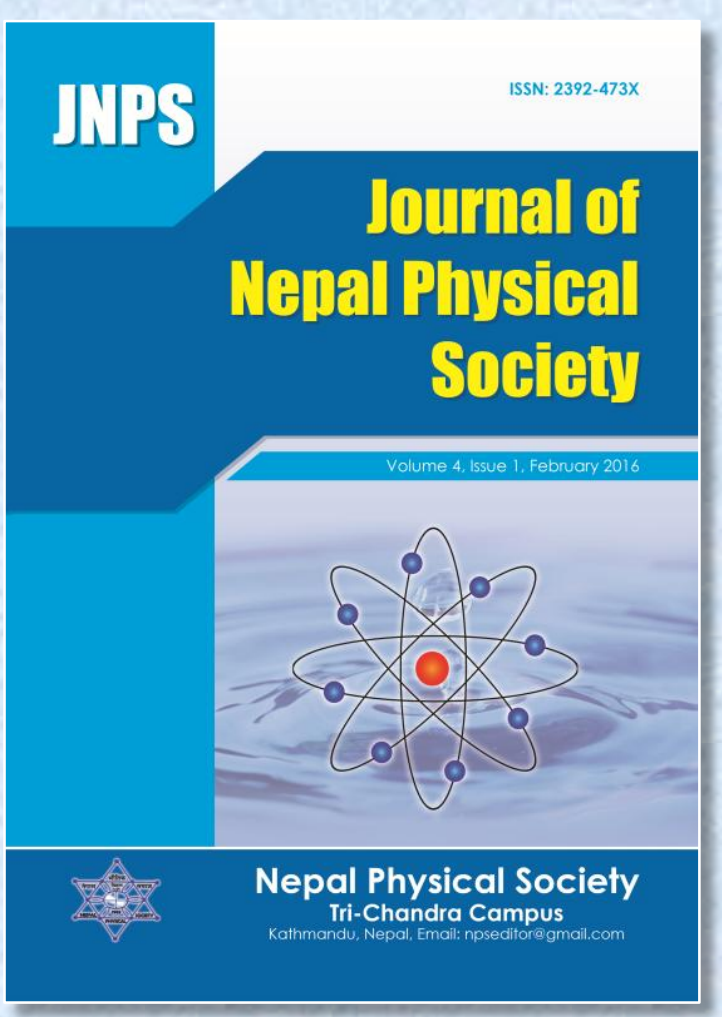

Published by:

Nepal Physical Society

P.O. Box : 2934

Tri-Chandra Campus

Kathmandu, Nepal

Email: npseditor@gmail.com 


\title{
Analysis of Geomagnetic Storms Using Wavelet Transforms
}

\author{
B. Adhikari ${ }^{1,2}$, R. Khatiwada ${ }^{1}$ and N. P. Chapagain ${ }^{2}$ \\ ${ }^{1}$ Department of Physics, St. Xavier's College, Maitighar, Kathmandu, Nepal \\ ${ }^{2}$ Departmentof Physics, Patan Multiple Campus, Tribhuvan University, Lalitpur, Nepal \\ ${ }^{*}$ Corresponding Email: binod.adhi@gmail.com
}

\begin{abstract}
Geomagnetic storms are recognized as a worldwide decrease of the horizontal component of the Earth's magnetic field measured at middle- and low-latitude magnetometers. The variations in the geomagnetic field during geomagnetic storms can be reproduced by changes in the interplanetary magnetic field and the solar wind velocity. Wavelet analysis, a useful statistical tool for analyzing variability has been used for this study. Wavelet transforms seem to be suited to analyze short-lived high-frequency phenomena such as discontinuities (shocks) in signals and transient structures. Wavelet analysis employed in the present work helps to decompose the time series of scales of interplanetary magnetic field (IMF-Bz) into different scales. To identify geomagnetic storms, the equatorial index, SYM-H has been used. The increase of wavelet coefficient amplitudes of the IMF-Bz is well correlated with the arrival of the shock and sheath region.
\end{abstract}

Keywords: Geomagnetic storms, Solar wind, Wavelet transform, Geomagnetic indices.

\section{INTRODUCTION}

The solar wind energy transfer to the magnetosphere and ionosphere is related to the solar activity. The activities of the sun are believed to be driven by energy release from the solar magnetic field (Gonzalez et al., 1994; Kamide et al., 1998). The solar activity on the Sun is not constant, and is closely related to the typical number of sunspots that are visible. The number of sunspots and the levels of solar activity vary with an 11 year period known as the solar cycle. The solar sources of the geomagnetic disturbances are known to have various interplanetary manifestations like coronal mass ejection (CME), magnetic clouds; co-rotating interaction region (CIR) and others interplanetary structures like interplanetary shocks (IPS), hemispheric current sheet (HCS) and sector boundary sector (SBS) etc. (Gonzalez et al., 1994; Burlaga, et al., 1981). These interplanetary medium, which often proves to be hostile conditions are controlled by the activity of the Sun, from which a constant flow of particles and magnetic field under special conditions may transfer energy and particles into the magnetosphere (Tsurutani et al., 2004; Laknina et al., 2006). The manifestations of these interactions are known geomagnetic disturbances. The major geomagnetic disturbances are refereed as storms and sub storms. During geomagnetic storms, several plasma regions are affected and occur strong modification. These modifications are caused by the mechanisms of viscous interaction, magnetic reconnection and resonant wave-particle interaction. The intensifications in the current systems mainly in the equatorial ring current are the main cause associated with these mechanisms. During the enhancement of equatorial ring current, particles acceleration and precipitation may occur in the auroral region, leading to aurora occurrences, which can cause telecommunications disturbances (Guarnieri et al., 2006).

The primary cause of storms is associated with plasma and magnetic field structure in the interplanetary medium. If the southward oriented $\mathrm{Bz}$ component of the interplanetary magnetic field (IMF) interconnect with the earth's magnetic field and sustained for enough time, we have the necessary conditions for a storm development (Gonzalez et al., 1994). The Bz with intensities higher than $10 \mathrm{nT}$ and sustained at least 3 hours is sufficient to cause an intense geomagnetic storm (Dst<-100 nT) (Gonzalez et al., 1994).

During solar maximum, the main structures emanating from the sun are erupting filaments and their associated Coronal Mass Ejection (CMEs) (Burlaga et.al, 1981). If the ICMEs coming from the sun into interplanetary space are faster enough (> $500 \mathrm{~km} / \mathrm{sec}$ ), i.e. relative velocity of solar wind is higher than the magneto-sonic speed (50-70 $\mathrm{km} / \mathrm{sec}$ ), a forward shock is formed. The sheath 
region, a strong shocked field can be found in the region between the shock and ICMEs. Another source of slowly varying and strong magnetic fields (10-25 nT) called as magnetic cloud can be found if the ICME has a well-organized magnetic field structure (Burlaga et.al, 1981). The interaction between these structures with the front of the magnetosphere caused compression of the magnetosphere due to the relative high density of the structures. It leads to an intensification of the magnetopause current, appearing as a positive sudden impulse in the Dst index. It is characteristic as storm sudden commencements (SSC), caused by the abrupt increase in the solar wind pressure at interplanetary shock. The positive impulse in the Dst index follows the SSC is storm initial phase, which may be of any length, from zero to more than 25 hours. However, not all geomagnetic storms have an initial phase and not all storm sudden commencement (SSC) is followed by a geomagnetic storm. In the main phase of the storm the horizontal component of the Earth's lowlatitude magnetic fields are significantly depressed by 100 (or more) nT and can be short as an hour or as long as a day. It is caused by a sustained southward interplanetary field reaching the magnetosphere. Long term sustained high amplitude $\mathrm{Bz}$ produces large particles injections into the magnetospheric radiation belt, known as Van-allen radiation belt. Such injection of particles produced a current encircling the earth called aring current. Ring current has diamagnetic nature and causes the decrease of the Dst index (Gonzalez et $a l .$, 1994). The recovery phase is the period where the field gradually recovers to the ambient value. During this phase, injected particles in the radiation belt start to dissipate, through several mechanisms (such as wave-particleinteractions, Coulomb scattering and Joule heating) and the Dst index slowly returnsto its ambient value.

\section{DATA SET}

In this work, two intense geomagnetic storm have been selected according to the Gonzalez et al. (1994) i.e. weak $(-50<$ Dst $\leq-30 \mathrm{nT})$, moderate $(-100<$ Dst $\leq$ $-50 \mathrm{nT})$, intense $(-250<\mathrm{Dst} \leq-100 \mathrm{nT})$, and very intense (Dst $\leq-250 \mathrm{nT})$. This dataset is compiled from the OMNI website https://omniweb.gsfc.nasa.gov/ form/dx1.html The SYM-H indices (1 $\mathrm{min}$ time resolution) used to identify the main phase of geomagnetic storm were also obtained from the same source. Interplanetary data sets used in this work are plasma speed (Vsw), density (Nsw), temperature
(Tsw) and magnitude of magnetic field $\left(\mathrm{B}_{\mathrm{mag}}\right)$ and components $(\mathrm{Bx}, \mathrm{By}, \mathrm{Bz})$. These datasets at $1 \mathrm{~min}$ time resolution were obtained also from the OMNI website. This site gives the high resolution OMNI datasets consisting of 1-min averaged ACE, WIND, IMP-8, Geo-tail and other solar wind data time-shifted to the bow shock.

\section{METHODOLOGY}

\section{Wavelet transform}

The basics idea of wavelet is small wave, localized wave and decay amplitude with respect to time/frequency. It cuts up data or functions in to different frequency components and then studies each component with a resolution match to its scale. A wavelet transform is a microscopic mathematical tool to "zoom in" short lived frequency phenomena such as discontinuities ("shocks") in signals. The wavelet transform is a transform that preserves the energy. The wavelet transform replaces the Fourier transform's sinusoidal waves by a family generated by translations and dilations of window called a wavelet.The transform of a signal evolving in time depends on two variables, scale (frequency) and time. It provides tool for time frequency localization.

The wavelet analysis is a mathematical technique which is very useful for numerical analysis and manipulation of multidimensional discrete signal sets. Originally, applied in geophysics to the analysis of seismic signals, the wavelet transforms were better and broadly formalized thanks to mathematicians, physicists, and engineers efforts (Morlet, 1983).

\section{Continuous wavelet transform (CWT)}

A continuous wavelet transform (CWT) is used to divide a continuous-time function into wavelets. It provides a very redundant and finely detailed description of a signal in terms of both time and frequency. Let ' $a$ ' and ' $b$ ' be the dilation and translation parameters that vary continuously over $\mathrm{R}$. Then the wavelet transform becomes

$$
\mathrm{W}(\mathrm{a}, \mathrm{b})=\int \mathrm{f}(\mathrm{t}) \Psi^{*}((\mathrm{t}-\mathrm{b}) / \mathrm{a}) \mathrm{dt}
$$

Where *represents the complex conjugate. This function $\mathrm{W}(\mathrm{a}, \mathrm{b})$ represents the wavelet coefficients. The variations of scale parameter give dilation effect when $a>0$ and contraction effect when $a<0$ of the mother wavelet function. So that it is possible to analyze the low and high frequency or the long and short period features of the signal.

\section{Waveletscalogram}

In signal processing, a scaleogram or scalogram is a 
visual method of displaying a wavelet transform.It represents the square amplitude of the modulus of the wavelet coefficients. It can be represented as the signal energy distribution in time $t$ and scale $a$.

\section{Discrete wavelet transform}

A discrete wavelet transform (DWT) is a wavelet transform in which the wavelets are discretely sampled. It contains discrete values of scale (j) and localization (k). So it may or may not have redundant representation depending on the Discretization scheme used (Daubechies, 1992; Domingues et al., 2005). In discrete wavelet transform the scale and the translation parameters give discrete values, i.e., $\mathrm{a}=2^{\mathrm{j}}$ and $\mathrm{b}=\left(2^{\mathrm{j}}\right) \mathrm{k}$. The discrete wavelet transform can be expressed in terms of wavelet coefficients as: $D_{k}^{j}=2^{-j / 2} \int f(t) \Psi^{j-}$ ${ }^{\mathrm{k}} \mathrm{dt} \quad$ where, $\Psi_{\mathrm{k}}^{\mathrm{j}}(\mathrm{t})=2^{-\mathrm{j} / 2} \Psi \quad\left(2^{-\mathrm{j}} \quad(\mathrm{t}-\mathrm{k})\right)$. The coefficients $\mathrm{d}_{\mathrm{k}}^{\mathrm{j}}$ are known as "details" (details are higher frequency structures or local approximation errors). For the purposes of our study, the Daubechies orthogonal wavelet of order two is chosen. A more detailed description of the wavelet technique can be found in Domingues et al. (2005), Mendes et al. (2005), Ojeda et al. (2014), Klausner et al. (2014a), Adhikari (2015), Adhikari and Chapagain (2016).

\section{RESULTS AND DISCUSSION}

Solar activity is directly related to space weather and geomagnetic activities rise and fall along with the solar activity. During geomagnetic storms, the Sun and magnetosphere are connected giving rise to several changes both in interplanetary space and terrestrial environment (Gonzalez et. al., 1994). Wavelet analysis employed in the present work helps to decompose the time series of solar wind and geomagnetic field parameters in to different scales. According to Gonzalez et al. (1994), geomagnetic storms can be classified as: weak, moderate, intense, and very intense as explained in section 2. Figure 1 shows solar wind parameters (Tp, Vsw, n/cc), magnitude of interplanetary magnetic field $\left(\mathrm{B}_{\mathrm{mag}}\right)$ and its components $(\mathrm{Bx}, \mathrm{By}$, $\mathrm{Bz}$ ) and geomagnetic indices SYM-H and $\mathrm{AE}$ during event on Febuurary 12-14, 2004. In this figure, the panels are from top to bottom, the solar wind temperature (Tp), the solar wind velocity (Vsw), the density (n/cc), the magnitude of interplanetary magnetic fields $\left(\mathrm{B}_{\mathrm{mag}}\right)$ and components $(\mathrm{Bx}, \mathrm{By}, \mathrm{Bz})$ and the geomagnetic indices SYM-H and AE. The storm to be occurred SYM-H must be $\leq-50 \mathrm{nT}$ ( Gonzalez et al.,1994) and density of solar wind plasma parcels, which appear to trigger super sub-storm event externally, about to lie between $\sim 30$ to $\sim 60 \mathrm{~cm}^{-3}$ (Tsurutani et al., 2015). Initial phase of this storm starts at the late day of 42 (February 11, 2004). The main phase starts around midnight and the long recovery phase last at the beginning of day 43 (February 11, 2004) to mid day of 46 (February 15, 2004).
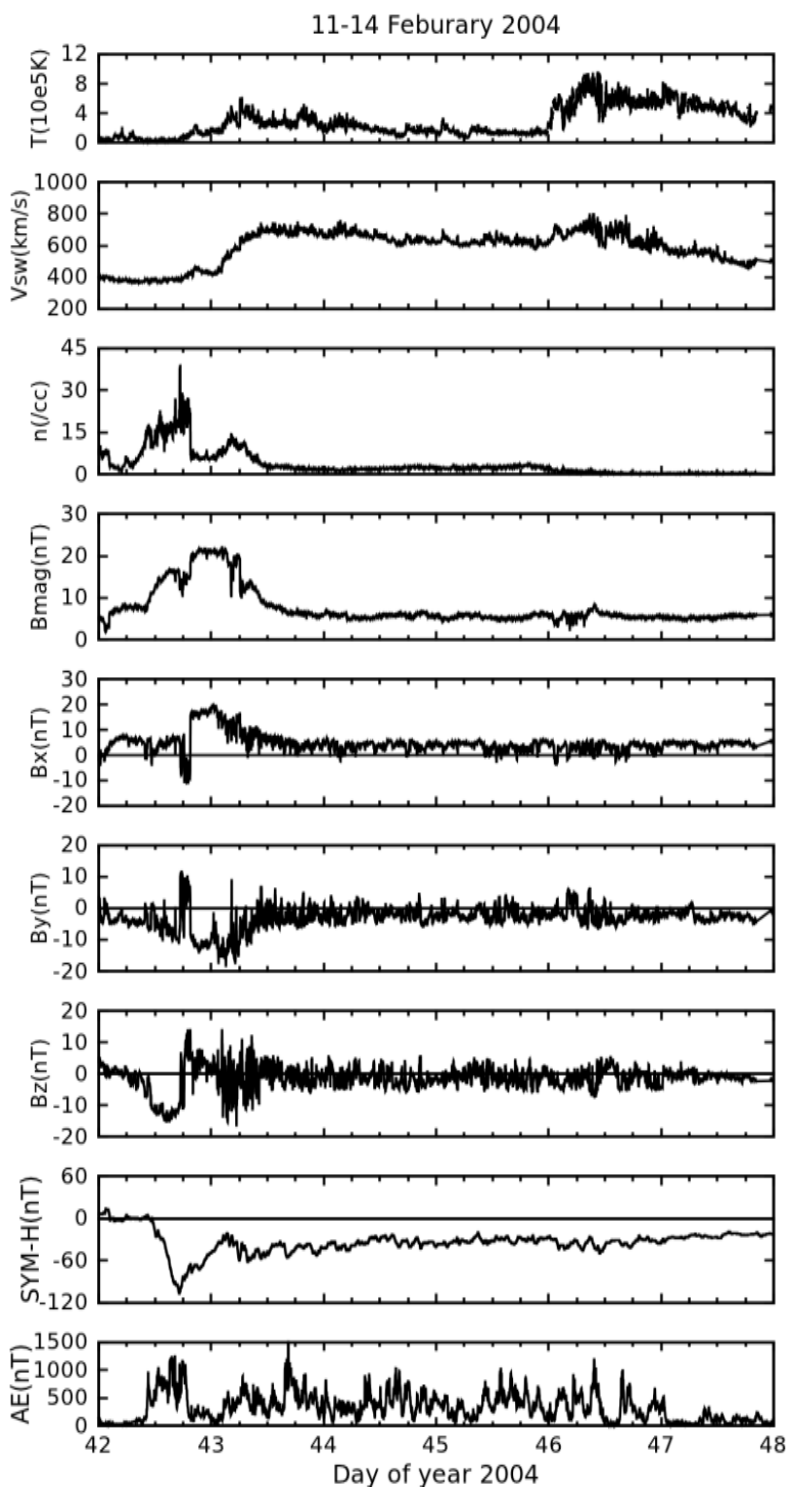

Fig. 1. Thepanel represents the interplanetary parameters during geomagnetic storm occurring on 11-14 February 2004. From top to bottom, the panel first shows solar wind temperature $T_{s w}$ $\left(10 e^{4} K\right)$, the second solar wind velocity $\left(V_{s w}\right.$ in $\mathrm{Km} / \mathrm{s})$, the third plasma parcel density $\left(n_{s w}\right.$ in $\left.\mathrm{cm}^{-3}\right)$, and the fourth panel for north-south component of the IMF (Bin $n T)$. The bottom panel scaled SYM-H (nT) and next to bottom panel contains the $A E$ index (nT) index. 
05 0ct 2000
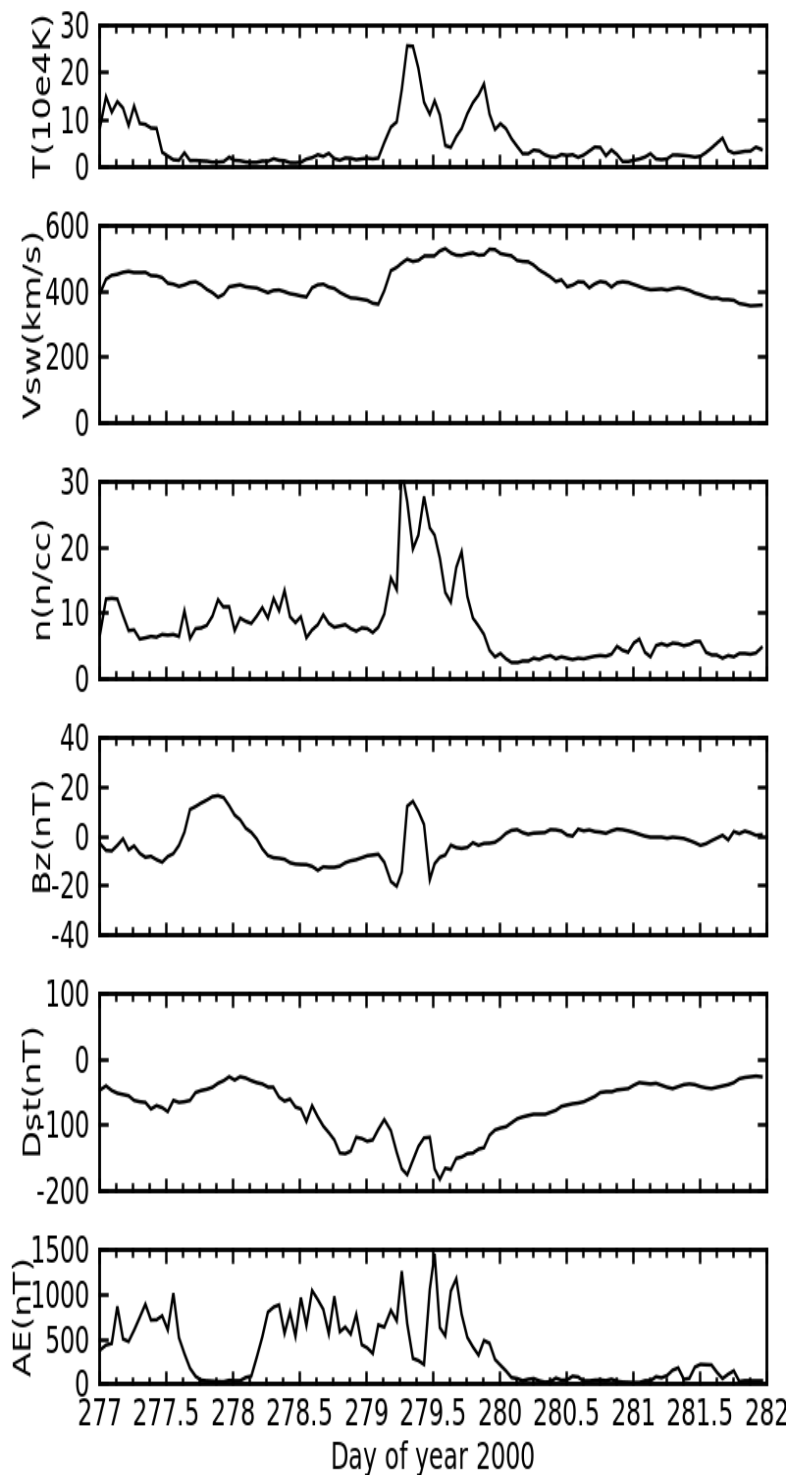

Fig. 2. From top to bottom, the panels shows the variations of the surface temperature (10e4K), solar wind plasma speed $\mathrm{Vsw}(\mathrm{km} / \mathrm{s})$, density (n/cc), south-north component of magnetic field $B z(n T)$ in GSM coordinate system, Dst (nT) and $A E(n T)$ indices with time (days) respectively of the Event, $5^{\text {th }}$ October 2000.

Similar type of the study is done for another event. Figure 2 shows the variation of solar wind parameters and geomagnetic indices (Dst and AE) with the day of the year of the event occurred on $5^{\text {th }}$ October, 2000. In the figure, top to bottom panel shows the solar wind speed around $600 \mathrm{~km} / \mathrm{s}$, plasma density is $30 \mathrm{cc}$, Dst shows more negative value of about $200 \mathrm{nT}$ indicating the selected storm as intense geomagnetic storm.

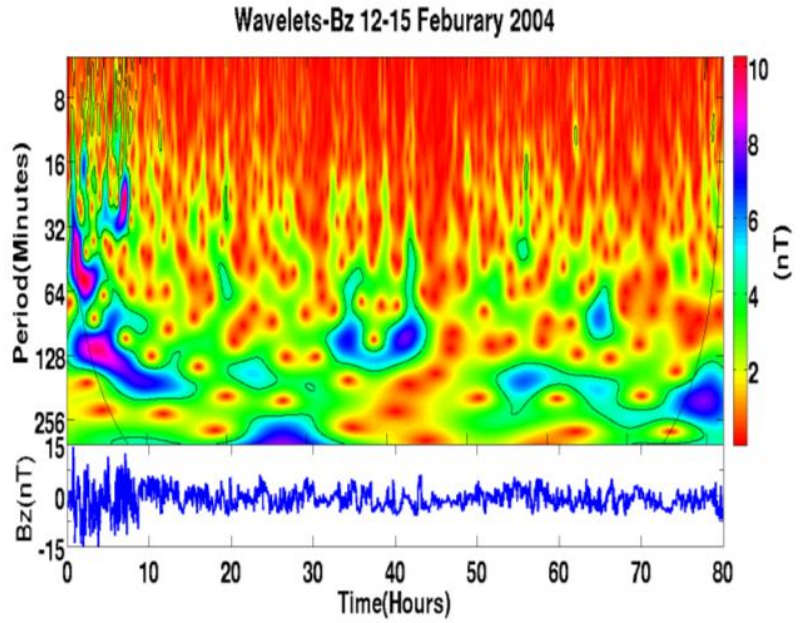

Fig. 3. Scalogram for Bz component during event on 12-15 February, 2004.

Figure 3 represents the result of scalograms of $\mathrm{Bz}$ during event on 12-15 Feb, 2005 . In the figure, the horizontal axis represents the time in hours and the vertical axis represents the periods in minutes. It shows the highest power areas around 2-10 hours at time scale approximately 20-128 minute. The another power areas covered by blue color are seen around 5-80 hours at time scales between 15-128 minutes. The less intense power areas also seen at different times and scales.

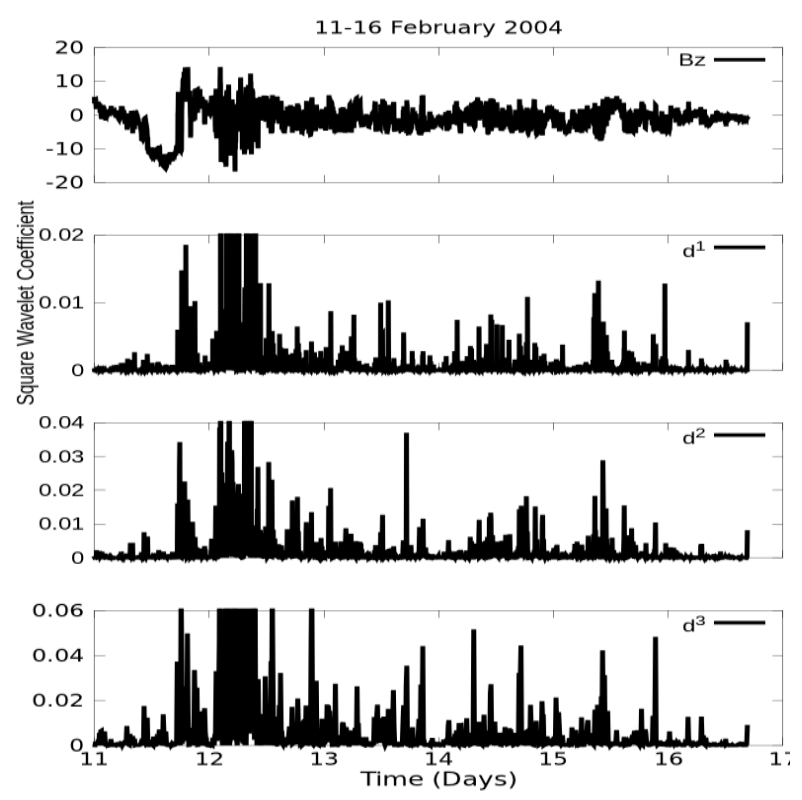

Fig. 4. Daubechies Wavelet coefficients dj (for $j=1,2,3, \ldots .7$ ) for Bz component during event occurred on 11-16 February, 2004. 
Figure 4 represents the results of discrete wavelet transform of $\mathrm{Bz}$ for the same event. The Daubechies order 2 orthogonal wavelet transform of seven levels $(j=1,2,3)$ has been used for this analysis. The seven levels $(j=1,2,3)$ are denoted by $\mathrm{d} 1, \mathrm{~d} 2, \mathrm{~d} 3$. For the chosen wavelet of frequency 0.66667 and sampling rate of one minute, the pseudo periods of the three levels were 3,6,12 minutes. The principle behind the use of the amplitude of the Daubechies wavelet coefficients is that they represent the local regularity present in the signal in study (Mendes et al., 2005; Ojeda et al., 2014). The highest amplitudes of the wavelet coefficients indicate singularities. The resulting singularity patterns is coincident with the main phase of the geomagnetic storms, which occur when large southward solar wind magnetic fields accompanied by the coronal mass ejection(CME) hit the Earth and severely disrupt the magnetosphere (Mendes et al., 2005; Adhikari et al., 2015, AdhikariChapagain, 2016).The highest amplitude of the square wavelet coefficients present at that time indicate singularities associated with storm. Whereas the smaller and larger amplitude of square wavelet coefficients indicated calm and disturbed conditions respectively. The main objective of this technique is to identify the sudden variation occur on $\mathrm{Bz}$ at the time of geomagnetic storm. The significant amplitude of square wavelet coefficients are seen for all levels. Observing these amplitude of the square wavelet coefficients, it can be noticed that $\mathrm{Bz}$ is severely affected at the time of geomagnetic storm.

\section{CONCLUSION}

We have presented a detail study of solar wind, interplanetary magnetic field during geomagnetic storm. The variation of solar wind parameters and geomagnetic indices has been studied taking south north component of interplanetary magnetic field $\mathrm{Bz}$ as the basis of the event selection. The results show that there were large disturbances due to presence of the solar and geomagnetic activities during the solar active period. During a geomagnetic disturbance, there is an energy input inside the magnetosphere, which changes atmospheric parameters, such as composition and temperature of the atmospheric particles. An important result that came out from the overall this analysis was that there exists a wavelet signature that clearly identifies the main phase of a geomagnetic storm period, for intense magnetic storms. The advantage of the time-frequency analysis method called Wavelet Transform resides in providing information not only about the frequencies of the event but also about its location in the time series.

\section{REFERENCES}

Adhikari, B. (2015). HILDCAA-Related Effects Recorded in Middle Low Latitude Magnetometers, InstitutoNacional de PesquisasEspaciais, Sao Jose dos Campos, Brasil.

Adhikari, B; Chapagain, N. P. (2015). Polar Cap Potential and Merging Electric Field during High Intensity Long Duration Continuous Auroral Activity, Journal of Nepal Physical Society, 3(1):6-17.

Burlaga, L. F.; Sittler, E.; Mariani, F. and Schwenn, R. (1981). Magnetic loop behind an interplanetary shock: Voyager, Helios and IMP 8 observations. Journal of Geophysical Research, 86:6673-6684.

Daubechies, I. (1992). Ten Lectures on Wavelets, CBMS-NSF Regional Conferene (Series in Applied Mathematics), vol. 61. SIAM, Philadelphia, PA.

Domingues, M. O.; Mendes, O. J. and Mendes, A. C. (2005). On wavelet techniques in atmospheric sciences Advances in Space Research, 35:831-842.

Guarnieri, F. F.; Tsurutani, B. T. and Gonzalez, W. D.; Gonzalez, A. L. C.; Grande, M.; Soraas, F.; Echer E. (2009). Icme and Cir storms with particular emphases on HILDCAA events, ILWS WORKSHOP 2006.

Gonzalez, W. D.; Joselyn, J. A.; Kamide, Y.; Kroehl, H. W.; Rostoker, G.; Tsurutani, B. T.; Vasyliunas, V. M. (1994): What is a geomagnetic storm qm, Journal of Geophysical Research, 99:5771-5792,

Klausner, V.; Domingues, M. O.; Mendes, O. and Papa, A. R. R. (2013). Tsunami effects on the $\mathrm{Z}$ component of the geomagnetic field, J.Atmos. Sol. Terr. Phys., 92:124-136.

Klausner, V.; Ojeda, G. A.; Domingues, M. O.; Mendes, O. and Papa, A. R. R. (2014). Study of local regularities in solar wind data and ground magnetograms, J. Atmos. Sol.-Terr. Phy., 112:10-19.

Kamide, Y.; Yokoyama, N.; Gonzalez, W. D.; Tsurutani, B. T.; Daglis, I. A.; Brakke, A.; 
Masuda, S. (1998). Two-step development of geomagnetic storms. Journal of Geophysical Research, 103:6917-6921.

Lakhina, G. S.; Alex, S.; Mukherjee, S.; Vichare, G. (2006). On magnetic storms and substorms. In: ILWS WORKSHOP 2006, GOA. [S.1.: s.n.].

Mendes, O. J.; Dominguesand, M. O.; Mendes, A. C. (2005). Wavelet analysis applied to magnetograms, Journal of Atmospheric and Solar Terrestrial Physics, 67:1827-1836.

Morlet, J. (1983). Sampling theory and wave propagation, Acoustic Signal/Image Processing and Recognition, (ed C. Chen), in NATO ASI. Springer-Verlag. New York, 1:233-261.

Ojeda, G. A.; Mendes, O.; Calzadilla, M. A. and Domingues, M. O. (2013). Spatio-temporal entropy analysis of the magnetic field to help magnetic cloud characterization, J. Geophys. Res., 118:5403-5414.
Ojeda, A.; Gonzalez, W. D.; Mendes, O.; Domingues, M. O. and Rosa, R. R. (2014). Nonlinear fluctuation analysis for a set of 41 magnetic clouds measured by the Advanced Composition Explorer (ACE) spacecraft, Nonlin. Processes Geophys., 21:1059-1073.

Tsurutani1, B. T.; Hajra, R.; Echer, E. and Gjerloev, J. W. (2015). Extremely intense (SML $\leq 2500$ nT) substorms: isolated events that are externally triggered? AnGeo Comm., 33:519-524. www.anngeophys.net/33/519/2015/ doi:10.5194/angeocom-33-519.

Tsurutani, B. T.; Gonzalez, W. D.; Guarnieri, F.; Kamide, Y.; Zhoua, X.; Arballo, J. K. (2004). Are high-intensity long-duration continuous AE activity (HILDCAA) events substorm expansion events qm. Journal of Atmospheric and Solar-Terrestrial Physics, 66:167-176. 